\title{
Investigación de Celdas de Combustible de Amoniaco Directo como fuente de Energía Alternativa. Desarrollo de Electrocatalizadores para las Reacciones de Electrodo en Medio Alcalino
}

\author{
Research on Direct Ammonia Fuel Cells as an Alternative Energy Source. \\ Development of a Low Temperature Fuel Cell Prototype
}

Presentación: 22/11/2019

\section{Doctorando:}

\section{Emir Esteban Saab}

Energías Alternativas, Tecnología y Desarrollo Sustentable (EnAITecS), Centro de Investigación y Desarrollo en Ciencia y Tecnología de Materiales (CITEMA), UTN, FRLP Avda. 60 y 124 S/N, Ciudad de Berisso, Pcia. Buenos Aires, Argentina, Tel: +54-221-412-4386, emirsaab@gmail.com.

\section{Director/es:}

Directora: Dra. Ana Castro Luna

Co-directores: Dr. Mariano Asteazaran y Dr. German Cespedes

\section{Resumen}

Las reservas de combustibles fósiles se consumen en cantidades crecientes y existe actualmente una gran preocupación en los gobiernos centrales por el grado de contaminación ambiental alcanzado durante las últimas décadas a nivel mundial. Disponer de energía es una necesidad en la vida moderna y poseerla se convertirá, en los próximos años, en un bien de alto valor en las sociedades desarrolladas. Las celdas de combustible son dispositivos que permiten la conversión directa de la energía química contenida en ciertos compuestos como el hidrógeno, metanol, etanol, amoníaco, en energía eléctrica. Las celdas de combustible alcalinas (AFCs) presentan una eficiencia mucho mayor que las que operan en medio ácido. A diferencia del metanol, etanol y etilenglicol, el amoníaco es un compuesto libre de carbono, por lo que no presenta como producto de reacción $\mathrm{CO}_{2}$, esto hace a el amoníaco un combustible ideal para las celdas con cero emisiones. Muchos investigadores ya han denominado al amoníaco como el combustible del futuro. La producción de amoníaco emplea como materia prima $\mathrm{N}_{2}$ del aire e $\mathrm{H}_{2}$ mediante un proceso bien conocido, sencillo y de bajo costo, empleando esencialmente el proceso Haber-Bosch. En nuestro país, se produce un excedente de $\mathrm{NH}_{3}$ de 80.000 ton/año, que mayormente se exporta. Desde el 2005, se han incrementado significativamente las publicaciones científicas con estudios detallados de la oxidación de

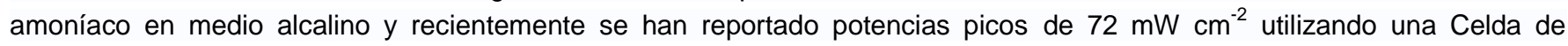
Combustible Alcalina de Amoníaco Directo (DAAFC). Esta propuesta de tesis pretende realizar aportes significativos en el área de energías alternativas y específicamente en el desarrollo de las DAAFCs. Se plantea como objetivo general desarrollar celdas alcalinas de amoniaco directo como una fuente de energía alternativa que trabaje a baja temperatura empleando membrana sólida intercambiadora de aniones.

En resumen, los objetivos específicos serán:

-Sintetizar y caracterizar física y electroquímicamente electrocatalizadores para la reacción de reducción de oxígeno (ORR). -Sintetizar y caracterizar física y electroquímicamente electrocatalizadores para la reacción de oxidación de amoníaco (AOR). -Desarrollar ensambles electrodo-membrana-electrodo (MEAs) con los catalizadores sintetizados en el laboratorio y evaluar física y electroquímicamente dichas MEAs.

-Desarrollar un prototipo de Celda de Combustible Alcalina de Amoniaco Directo (DAAFC).

Palabras claves: celda de combustible energías alternativas tecnología del hidrogeno 


\section{Abstract}

Fossil fuel reserves are consumed in increasing quantities and there is currently a great concern in central governments about the degree of environmental pollution affected during the last decades worldwide. Having energy is a necessity in modern life and possessing it will become, in the coming years, a good of high value in developed societies. Fuel cells are devices that allow the direct conversion of chemical energy contained in certain compounds such as hydrogen, methanol, ethanol, ammonia, into electrical energy. Alkaline fuel cells (AFC) have a much higher efficiency than those operating in an acid medium. Unlike methanol, ethanol and ethylene glycol, ammonia is a carbon-free compound, so it is not presented as a $\mathrm{CO}_{2}$ reaction product, this makes ammonia an ideal fuel for cells with zero emissions. Many researchers have already described ammonia as the fuel of the future. Ammonia production uses $\mathrm{N}_{2}$ of the air and $\mathrm{H}_{2}$ as a raw material through a well-known, simple and low-cost process, specifically using the Haber-Bosch process. In our country, we produce a surplus of $\mathrm{NH}_{3}$ of 80,000 tons / year, which is mostly exported. Since 2005, scientific publications have been increased with detailed studies of ammonia oxidation in alkaline medium and recently peak powers of $72 \mathrm{~mW} \mathrm{~cm}^{-2}$ have been reported using a Direct Ammonia Alkaline Fuel Cell (DAAFC). This thesis proposal aims to make specific contributions in the area of alternative and specific energies in the development of DAAFCs. The general objective is to develop direct ammonia alkaline cells as an alternative energy source that works at a low temperature using anion exchange solid membrane. In summary, the specific objectives will be:

-Synthesize and characterize physically and electrochemically electrocatalysts for the oxygen reduction reaction (ORR).

-Synthesize and characterize physically and electrochemically electrocatalysts for the reaction of ammonia oxidation (AOR).

-Develop electrode-membrane-electrode (MEAs) assemblies with the catalysts synthesized in the laboratory and evaluate said MEAs physically and electrochemically.

-Develop a prototype of Direct Ammonia Alkaline Fuel Cell (DAAFC).

Keywords: fuel cell alternative energies hydrogen technology

\section{Introducción}

El objetivo general de la tesis es desarrollar celdas alcalinas de amoniaco directo como una fuente de energía alternativa, que trabaje a baja temperatura empleando membrana sólida intercambiadora de aniones.

Los objetivos específicos son los mencionados anteriormente en el resumen, que para llevarlos a cabo es necesario emplear una serie de métodos y técnicas, las cuales serán mencionadas a continuación:

- $\quad$ Sintetizar electrocatalizadores para la reacción de reducción de oxígeno (ORR)

La actividad catalítica de los materiales a preparar depende fuertemente del método de síntesis a implementar, ya que éste puede controlar el tamaño, forma y composición de los nanopartículas catalíticas.

Se desarrollarán nuevos electrocatalizadores multicomponentes del tipo PtM, AgM y PdM (con M= Cu, $\mathrm{Au}, \mathrm{Sn}, \mathrm{Ni}, \mathrm{Mg}$, etc.) dispersados sobre un soporte conductor de alta área superficial $\left(\mathrm{C}, \mathrm{Mn}_{3} \mathrm{O}_{4}\right.$, óxido de grafeno, etc.) empleando métodos de síntesis que involucran la reducción química de los precursores metálicos para el catalizador en cuestión, empleando agentes surfactante para controlar el tamaño y forma de la nanopartícula y como agente reductor etilenglicol (método poliol), borohidruro de sodio, $\mathrm{H}_{2} / \mathrm{N}_{2}$ a altas temperatura, etc. [Qaseem 2016, Shao 2016, Park 2015].

En general, la metodología de síntesis química, con agentes reductores, implica la homogeneización en baño de ultrasonido de la mezcla de los precursores metálicos, el soporte conductor y el agente reductor, con variaciones del pH del medio para mejorar el anclaje de los iones metálicos al soporte conductor, un calentamiento a reflujo por el término de al menos $3 \mathrm{~h}$, seguido de una etapa de reposo, aislamiento, lavado del sólido y secado en estufa por $12 \mathrm{~h}$ a $60{ }^{\circ} \mathrm{C}$ [Chen 2005]. El calentamiento tradicional a reflujo puede reemplazarse por calentamiento asistido por microondas lo que reduce a minutos el proceso de síntesis [Asteazaran 2014].

- Caracterizar física y electroquímicamente los materiales sintetizados para la ORR

Caracterización Fisicoquímica

Por microscopía de transmisión de alta resolución (HRTEM) se determinará el tamaño de partícula y distribución de las mismas sobre el soporte. La composición superficial y el estado de oxidación de los diferentes componentes de los catalizadores, así como de los soportes usados, se determinarán por espectroscopia de fotoelectrones de rayos X (XPS). La composición volumétrica de las muestras se llevará a cabo por, espectroscopia con energía dispersiva de rayos X (EDX) y la posible formación de aleaciones y el estado cristalino se determinará con el uso de difracción de rayos X en polvos (XRD) [Van Cleve 2016, Asteazaran 2014]. 
Caracterización Electroquímica

El comportamiento de los catalizadores sintetizados para la Reacción de Reducción de Oxígeno (ORR) se estudiará utilizando la técnica de Electrodo de Disco Rotante de capa delgada (RDE). Ésta consiste en un disco de carbón vítreo sobre el que se deposita el catalizador sintetizado y se lo cubre con una película de polímero conductor de aniones, de este modo se simulan las condiciones que se tienen en las celdas de combustible. Las determinaciones se realizarán en una celda electroquímica convencional de tres electrodos. Se empleará como electrodo de trabajo un RDE, como contraelectrodo una lámina de Pt, como electrodo de referencia un electrodo de calomelanos (SCE) y el electrolito será una solución $\mathrm{KOH} 1 \mathrm{M}$ saturada con $\mathrm{N}_{2}$. Se llevarán a cabo técnicas electroquímicas como barrido lineal de potencial, voltamperometría cíclica, espectroscopia de impedancia electroquímica, cronoamperometría, entre otras, con el objetivo de determinar la actividad catalítica de los materiales sintetizados. Adicionalmente se estudiará el comportamiento de los catalizadores a tiempos prolongados de operación para determinar los catalizadores óptimo a utilizar en los ensambles MEAs de la celda decombustible de amoniaco directo (DAAFC).

- $\quad$ Sintetizar electrocatalizadores para la reacción de oxidación de amoníaco (AOR)

El método de síntesis utilizado, para obtener catalizadores para la electrooxidación de amoniaco, juega un papel muy importante en la performance de los materiales activos producidos. Las propiedades físicas y químicas del material sintetizado (composición, grado de aleación, tamaño de partícula, estructura superficial, morfología, etc.) dependen fuertemente del método de síntesis empleado.

Se han desarrollado numerosos métodos de síntesis para la preparación de nanopartículas catalíticas, siendo los métodos de reducción química los más empleados; donde los precursores metálicos se dispersan en un solvente adecuado y se reducen con etilenglicol, $\mathrm{NaBH}_{4}, \mathrm{H}_{2}$, etc. Normalmente se agrega un agente estabilizante el cual se adsorbe sobre la superficie de la nanopartícula evitando la aglomeración de las partículas. Se sintetizarán electrocatalizadores multicomponente en base Pt y Pd del tipo PtM y PdM con M=Ir, Ni, Pd, SnOx, $\mathrm{Au}, \mathrm{Ru}, \mathrm{Rh}$, por reducción química de las sales de los precursores metálicos para la obtención del catalizador deseado [Silva 2015, Assumpção 2015, Vidal-Iglesias 2007, Matsuoka 2005]. De forma semejante a lo descrito en la síntesis para electrocatalizadores para la ORR, se emplearán métodos de síntesis química tales como poliol con etilenglicol como agente reductor [Lomocso 2008, Tao 2008] o método de microemulsión usando borohidruro de sodio como reductor químico [Ganguli 2010, Boutonnet 2008, Vidal-Iglesias 2007]. En el diseño de los catalizadores para la electrooxidación de amoniaco en medio alcalino se tendrá en cuenta la composición de los catalizadores deseados, la relación atómica más adecuada entre los elementos que conformarán el catalizador, la estructura y tamaño de las nanopartículas, la carga de material activo sobre el soporte conductor, etc. [Silva 2015, Zhong 2013, Endo 2004].

\section{- Caracterizar física y electroquímicamente los materiales sintetizados para la AOR}

La performance electrocatalítica de los materiales sintetizados para la AOR se caracterizan normalmente por una serie de parámetros que incluyen la i) Actividad Másica (MA) que es la densidad de corriente normalizada por la masa de electrocatalizador medida a un potencial específico, ii) Actividad Específica (SA) que representa la densidad de corriente normalizada por el Área Superficial Electroquímicamente Activa (ECSA) del electrocatalizador. Para la mayoría de los metales nobles, el ECSA puede ser medido por la carga involucrada en la región de adsorción-desorción de hidrógeno usando el valor correspondiente a la carga específica para la adsorción de una monocapa de hidrógeno (por ejemplo, para Pt, Ir, Pd y Rh, suele usarse $210,218,212$ y $221 \mu \mathrm{C} \mathrm{cm}^{-2}$ respectivamente), iii) durabilidad, es decir la habilidad del electrocatalizador a resistir cambios permanentes de la performance en el tiempo [Katayama 2015, Zhong 2013].

Los estudios electroquímicos de los catalizadores para la AOR se llevarán a cabo en una celda convencional de vidrio de tres electrodos, empleando como electrodo de trabajo un electrodo de disco rotante de carbón vítreo sobre el que se depositara el catalizador sintetizado, una chapa de Pt de $1 \mathrm{~cm}_{2}$ como contraelectrodo y un electrodo de calomelanos sat. (SCE) como electrodo de referencia. Se utilizará como electrolito $\mathrm{KOH} 1 \mathrm{M}$ y una solución de $\mathrm{NH}_{3} 0,1 \mathrm{M}$ en $\mathrm{KOH} 1 \mathrm{M}$. Las mediciones se llevarán a cabo a temperatura ambiente previa desoxigenación con N2 de la solución electrolítica.

En la determinación de la performance electroquímica se emplearán técnicas de voltamperometría lineal y cíclica, cronoamperometría, espectroscopia de impedancia electroquímica.

La caracterización física se llevará a cabo al igual que en el punto (3) de la metodología empleando microscopía de transmisión de alta resolución (HRTEM), espectroscopia de fotoelectrones de rayos X (XPS), espectroscopia con energía dispersiva de rayos $X(E D X)$ y difracción de rayos $X$ en polvos (XRD).

- Desarrollar ensambles electrodo-membrana-electrodo (MEA) con los catalizadores comerciales y con los desarrollados en el laboratorio

La MEA es la parte fundamental de la celda de combustible, está compuesta de dos electrodos, ánodo y cátodo, separados por una membrana conductora de aniones. En el transcurso del desarrollo de la tesis se prepararán MEAs de cinco capas mediante la técnica de Catalyst Coated Membrane (CCM): capa difusora y capa catalítica anódicas, membrana de intercambio aniónica (AEM) y capa catalítica y difusora catódica [Kocha S.S. 2003]. La CCM consiste en depositar una capa catalítica (CL) catódica sobre uno de los lados de la AEM. Sobre dicha CL se coloca una la capa difusora de gases (GDL) para facilitar la difusión del $\mathrm{O} 2$ por el electrodo poroso hasta alcanzar, de forma uniforme, los sitios activos de la CL. Sobre el otro lado de la 
AEM se realiza de forma similar el depósito de la capa catalítica anódica con su correspondiente capa difusora. Las capas catalíticas están constituidas principalmente por las nanopartículas catalíticas soportadas y el ionómero conductor de aniones. Se preparará la CL con los catalizadores sintetizados en el laboratorio, así como con catalizadores comerciales cuyo comportamiento electrocatalíticas podrá ser utilizado como referencia.

La capa difusora de gases consiste de dos subcapas, una macroporosa (GDL) y una microporosa (MPL). Para la GDL se empleará una tela de carbón de alta conductividad la cual será sometida a un tratamiento para mejorar su hidrofobicidad. Para este tratamiento se sumergirá la tela de carbón en una suspensión de Teflón ${ }^{\circledR}$ y posteriormente se secará la misma en estufa a $80^{\circ} \mathrm{C}$. Esta operación se repetirá hasta lograr una concentración adecuada de Teflón®. Para la MPL se ultrasonicará durante 2 h una mezcla de negro de acetileno, suspensión de Teflón® al 60 \%wt y alcohol isopropílico. Esta suspensión será aplicada sobre la tela de carbón hidrofóbica mediante la técnica de doctor-blade en una proporción de $2 \mathrm{mg} \mathrm{cm}^{-2}$ de negro de acetileno. Como AEM se empleará la membrana comercial desarrollada por Tokuyama Co. (Tokyo, Japón). Para su utilización en la DAAFC se debe primero funcionalizar para que sea capaz de conducir iones $\mathrm{OH}$.

- Evaluar física y electroquímicamente las MEAs en una celda de tres electrodos.

La performance de las MEAs se estudiarán en una celda electroquímica convencional de tres electrodos donde el electrodo de trabajo será una pequeña porción de MEA colocado en un dispositivo especial de Teflón® que ha sido desarrollada en el laboratorio [Asteazaran 2015C]. Esta celda de Teflón ${ }^{\circledR}$ permite estudiar ensambles de pequeñas dimensiones y extrapolar los resultados a ensambles de mayor tamaño. La performance depende de las condiciones de operación: temperatura de trabajo, tipo de oxidante (O2 o aire), presión de los gases, velocidad de flujo de los reactivos, etc. y de las características de los componentes de la celda: carga del catalizador, procedimiento de fabricación de los electrodos, acondicionamiento de la MEA, conductividad de la membrana, etc. [Cao X. 2012, Carton J.G. 2010].

Se realizará la caracterización electroquímica de las MEAs mediante técnicas tales como barridos lineales de potencial, cronoamperometría, espectroscopia de impedancia electroquímica y la caracterización física se realizará con el auxilio de técnicas tales como microscopía de transmisión electrónica de alta resolución HDTEM, espectroscopia de dispersión de electrones de rayos $X$ (EDS), espectroscopia de fotoelectrones de rayos $X$ (XPS), difracción de rayos $X$ (XRD), análisis termogravimétrico (TGA), etc., técnicas que ya se mencionaron anteriormente.

- $\quad$ Desarrollar un prototipo de Celda de Combustible de Amoniaco Directo (DAAFC)

Como parte final de la tesis se pondrá a prueba las MEAs desarrolladas en el laboratorio en un prototipo de celdas de combustible alcalina de amoníaco directo. En ésta etapa se utilizarán las partes componentes de una celda de combustible que se dispone en el laboratorio que cumple con las condiciones necesarias para el estudio. Para esta celda se desarrollarán MEAs de $5 \mathrm{~cm} 2$ de área geométrica. Estas MEAs se realizarán con los catalizadores sintetizados en el laboratorio que hayan presentado la mejor performance. Las mediciones se realizarán mediante el auxilio de un potenciostato Gamry Reference 3000. El cátodo de la celda será alimentado tanto con $\mathrm{O}_{2}$ de alta pureza como con aire, para evaluar la performance de la celda de combustible en ambas condiciones.

\section{Resultados}

El platino es el catalizador más activo para el proceso de oxidación de amoníaco, sin embargo, es caro y se inactiva fácilmente por la adsorción de nitrógeno. Se han investigado diversos catalizadores mono y bimetálicos. Para hacer que las FC de amoniaco sean comercialmente atractivos, la cantidad de metales preciosos debe reducirse. Esto se puede lograr utilizando electrocatalizadores como nanopartículas dispersas en soportes conductores de alta área de superficie, por ejemplo, negro de carbón.

Hasta el momento, se sintetizaron catalizadores bimetálicos soportados sobre negro de carbón, PtM y PtMRu ( $\mathrm{M}=\mathrm{Co}, \mathrm{Cu}$, Ru, $\mathrm{Ni}$, Ir) trimetálicos utilizando un método de impregnación modificado. Estos materiales sintetizados han sido estudiados como potenciales catalizadores para la electrooxidación de amoníaco.

Técnicas como voltamperometría cíclica (CV), cronoamperometría (CA) y espectroscopia de impedancia electroquímica (EIS) se utilizaron para la caracterización electroquímica. Todas las mediciones electroquímicas se llevaron a cabo en una célula de PTFE de tres electrodos. Esta celda electroquímica de PTFE fue diseñara y desarrollada en el marco de la tesis doctoral.

Se utilizaron una gran superficie de $\mathrm{Pt}$ y un electrodo de $\mathrm{Ag} / \mathrm{AgCl}$ como contraelectrodo y electrodo de referencia, respectivamente. Como electrodo de trabajo se usó un carbono vítreo con una capa delgada de catalizador. Se evaluó el comportamiento de los nuevos materiales frente a la electrooxidación de amoníaco. Se utilizó KOH $1 \mathrm{M}$ como electrolito soporte, al que se agregaron cantidades crecientes de $\mathrm{NH}_{4} \mathrm{OH}$. Los catalizadores sintetizados han demostrado ser muy atractivos como materiales activos para el ánodo de una celda de combustible de amoniaco directo. La adición de cocatalizadores al Pt ha mejorado su comportamiento electrocatalítico hacia la electrooxidación de amoníaco.

Brevemente, el método de síntesis consiste en impregnar polvo de carbón (Vulcan $\AA$ ) con una solución de los precursores metálicos (por ejemplo $\mathrm{H}_{2} \mathrm{PtCl}_{6}, \mathrm{RuCl}_{3}, \mathrm{CuSO}_{4}, \mathrm{IrCl}_{3}$, etc.) a depositar y realizar la reducción con un agente químico adecuado. 
Las mediciones electroquímicas se llevaron a cabo a temperatura ambiente utilizando un potenciostato Gamry Reference $3000^{\circledR}$. Debido a alta concentración de álcali con la que se trabaja, se diseñó y construyó en el laboratorio la celda electroquímica de tres electrodos de PTFE antes mencionada. Como electrodo de trabajo se utilizó un electrodo de disco rotante (RDE) de carbón vítreo $\left(0,07 \mathrm{~cm}^{2}\right)$, sobre el que se depositó el electrocatalizador en estudio. Se utilizaron un electrodo de Pt como contra-electrodo y uno de $\mathrm{Ag} / \mathrm{AgCl}$ como electrodo de referencia $(\mathrm{E}=0,22 \mathrm{~V})$.

Para la preparación del electrodo de trabajo, se pulió el carbón vítreo con una suspensión de alúmina y se lavó con agua desionizada. Se utilizó agua ultrapura obtenida de un sistema Milli- $Q^{\circledR}$ en todos los procedimientos experimentales. La tinta catalítica se preparó dispersando $6 \mathrm{mg}$ del polvo del electrocatalizador en agua. La misma se ultrasonicó en un baño durante 30 min. Posteriormente, se depositaron $12 \mu \mathrm{l}$ del dispersado sobre la superficie del RDE y una vez seco se adicionaron $84 \mu \mathrm{l}$ de solución de Nafion $^{\circledR}$ al $5 \%$ en metanol. Los materiales sintetizados fueron evaluados electroquímicamente para determinar su desempeño. Se empleó como referencia el comportamiento de un catalizador comercial de Pt (E-TEK $\left.{ }^{\circledR}\right)$.

Para la caracterización electroquímica se utilizaron técnicas CV, CA y EIS. Se utilizó una solución de hidróxido de potasio como electrolito soporte, al que se agregaron cantidades crecientes de una solución concentrada de $\mathrm{NH}_{3}$.

Se realizaron voltamperometrías cíclicas a una velocidad de barrido de $20 \mathrm{mVs}^{-1}$ entre -0.05 hasta $1,1 \mathrm{~V}$ en $\mathrm{KOH}_{1} \mathrm{M}$ como electrolito soporte y concentraciones de $0,01-0,5 \mathrm{M}$ de $\mathrm{NH}_{4} \mathrm{OH}$. Los electrocatalizadores fueron previamente

ciclados en $\mathrm{KOH} 1 \mathrm{M}$ hasta obtener un perfil estable. Los experimentos crono-amperométricos se llevaron a cabo durante $600 \mathrm{~s}$ a $\mathrm{E}=0,35 \mathrm{~V}$. En los gráficos las densidades de corriente se normalizaron respecto a la carga de Pt.

Los materiales sintetizados han demostrado un buen desempeño para la electro-oxidación de amoníaco.

\section{Referencias}

Assumpção M.H.M.T., Piasentin R.M., Hammer P., Rodrigo De Souza F.B, Buzzo G.S., Santos M.C.

Asteazaran M, Bengió S, Triaca W.E., Castro Luna A.M. "Methanol tolerant electrocatalysts for the oxygen reduction reaction" Journal of Appl Electrochemistry 44 (2014), 1271-1278.

Asteazaran M, Bengió S, Triaca W.E., Castro Luna A.M. "Methanol tolerant electrocatalysts for the oxygen reduction reaction". Journal of Appl Electrochemistry (2014) 44, 1271-1278. doi 10.1007/s10800-014-0748-1.

Asteazaran M. , Cespedes G. , Bengió S. , Moreno M.S. , Triaca W.E. , Castro Luna A.M. "Research on methanol tolerant catalysts for the oxygen reduction reaction”. Journal of Applied Electrochemistry (2015). doi 10.1007/s10800-015-0845-9.

Asteazaran M., Cespedes G., Moreno M.S., Bengio S., Castro Luna A.M.. "Searching for Suitable Catalysts for a Passive Direct Methanol Fuel Cell”. Cathode Int. J of Hydrogen Energy (2015) doi 10.1016/j.ijhydene.2015.05.134.

Asteazaran M., Cespedes G., Castro Luna A.M. "Investigación de la Actividad Catalítica de Material de Electrodo para Celda de Combustible en Electrodo de Disco Rotante y Electrodo de Difusión de Gases". III Congreso Argentino de Ingeniería Química (CAIQ2015). Buenos Aires. 2-5 Agosto 2015.

Boutonnet M., Lögdberg S., Svensson E.E. "Recent developments in the application of nanoparticles prepared from w/o microemulsions in heterogeneous catalysis". Colloid Interface Sci., 2008, 13, 270-286.

Endo K, Nakamura K, Katayama Y, Miura T. "Pt-Me (Me = Ir, Ru, Ni) binary alloys as an ammonia oxidation anode". Electrochimica Acta 49 (2004) 2503-2509.

Ganguli A.K., Ganguly A., Vaidya S. "Microemulsion-based synthesis of nanocrystalline materials". Chem. Soc. Rev., 2010, 39, 474-485.

Katayama Y, Okanishi T, Muroyama H, Matsui T, Eguchi K. "Electrochemical Oxidation of Ammonia over Rare Earth Oxide Modified Platinum Catalysts". J. Phys. Chem. C, 119 (2015) 9134-9141. doi: 10.1021/acs.jpcc.5b01710

Lomocso T.L., Baranova E.A. "Electrochemical oxidation of ammonia on carbon-supported bi-metallic PtM (M = Ir, Pd, SnO x ) nanoparticles". Electrochim. Acta, 56 (2011) 8551-8558. Pág. 19 / 20

Matsuoka K, Iriyama Y, Abe T, Matsuoka M, Ogumi Z. "Alkaline direct alcohol fuel cells using an anion exchange membrane". Journal of Power Sources 150 (2005) 27-31.

Qaseem A, Chen F, Wu X, Johnston R.L. "Pt-free Silver Nanoalloy Electrocatalysts for Oxygen Reduction Reaction in Alkaline Media”. Catal. Sci. Technol., (2016) Accepted Manuscript.

Shao M, Chang Q, Dodelet J, Chenitz R. "Recent Advances in Electrocatalysts for Oxygen Reduction Reaction". Chem. Rev. (2016) Accepted Manuscript.

Silva J.C.M., da Silva S.G., De Souza R.F.B., Buzzo G.S., Spinacé E.V., Neto A.O., Assumpcão M.H.M.T.. "PtAu/C electrocatalysts as anodes for direct ammonia fuel cell”. Applied Catalysis A: General 490 (2015) 133-138.

Van Cleve T. "Development of cathodic electrocatalysts for low temperature H2 fuel cell applications: Improving oxygen reduction activity through the manipulation of size, shape, and composition". Tesis Doctoral (2016) University of Michigan. 
Vidal-Iglesias F.J., Solla-Gullon J., Montiel V., Feliu J.M., Aldaz A. "Screening of electrocatalysts for direct ammonia fuel cell: Ammonia oxidation on PtMe (Me: Ir, Rh, Pd, Ru) and preferentially oriented Pt(1 00 ) nanoparticles". Journal of Power Sources 171 (2007) 448-456.

Zhong C., Hu W.B., Cheng Y.F.. "Recent advances in electrocatalysts for electro-oxidation of ammonia". Journal of Materials Chemistry A (2013). 